\title{
Comparative efficacy, acceptability, and tolerability of lisdexamfetamine in child and adolescent ADHD: a meta-analysis of randomized, controlled trials
}

This article was published in the following Dove Press journal:

Drug Design, Development and Therapy

I April 2015

Number of times this article has been viewed

\author{
Benchalak Maneeton' \\ Narong Maneeton' \\ Surinporn Likhitsathian' \\ Sirijit Suttajit' \\ Assawin Narkpongphun' \\ Manit Srisurapanont' \\ Pakapan Woottiluk ${ }^{2}$ \\ 'Department of Psychiatry, Faculty \\ of Medicine, Chiang Mai University, \\ Chiang Mai, Thailand; ${ }^{2}$ Psychiatric \\ Nursing Division, Faculty of Nursing, \\ Chiang Mai University, Chiang Mai, \\ Thailand
}

Background: Several studies have shown that lisdexamfetamine (LDX) is efficacious in children and adolescents with attention-deficit/hyperactivity disorder (ADHD).

Objectives: Aims of this study were to systematically review the efficacy, acceptability, and tolerability of LDX in child and adolescent ADHD. Any randomized controlled trials (RCTs) of LDX versus placebo carried out in children and adolescents with ADHD were included.

Data sources: The searches of the SCOPUS, MEDLINE, CINAHL and Cochrane Controlled Trials Register were performed in September 2014. Additional searches in the ClinicalTrials. gov and EU Clinical Trials Register database were conducted.

Study eligibility criteria, participants, and interventions: This review included all RCTs of LDX versus placebo which were carried out in children and adolescents up to 18 years old. Additionally, the included studies must have reported the final outcomes of: i) severity of ADHD symptoms with standardized scales, ii) rates of improvement, iii) rates of discontinuation. To be more thorough, the languages of such RCTs were not limited.

Study appraisal and synthesis methods: The abstracts from databases were inspected and the full text versions of relevant trials were examined and extracted for important outcomes. The efficacious measurements included either the pooled mean end-point or changed scores of ADHD rating scales, and the rate of improvement. Acceptability and tolerability were measured by the pooled overall discontinuation rate and the pooled discontinuation rate due to adverse events, respectively. A random effect model technique was utilized to synthesize the mean differences (either standardized mean differences or weighted mean differences) and relative risks (RRs) with 95\% confidence intervals (CIs).

Results: A total of 1,016 children and adolescents with ADHD were included. The dosage of LDX was 30 to $70 \mathrm{mg}$ /day. The pooled mean change scores of LDX-treated group was significantly greater than that of the placebo (weighted mean difference $[95 \% \mathrm{CI}]$ of -15.20 $[-19.95,-10.46], I^{2}=94 \%$ ). The pooled improvement rate of the LDX-treated group was also significantly higher than that of the placebo (RR $[95 \% \mathrm{CI}]$ of $0.34[0.24,0.47], I^{2}=80 \%$ ). The pooled overall discontinuation rate between the two groups was not significantly different (RR [95\% CI] of $0.78[0.46,1.31], I^{2}=63 \%$ ). Similarly, the pooled discontinuation rate due to adverse events between the two groups showed no significant difference (RR [95\% CI] of 1.99 $\left.[0.70,5.64], I^{2}=0 \%\right)$.

Limitations: The number of included studies was limited (five RCTs).

Conclusion: According to the present review, LDX was effective and well-tolerated in the treatment of child and adolescent ADHD. Unfortunately, the acceptability of LDX was not better than the placebo. Since the number of included studies was limited, the outcome from this review should be carefully interpreted and considered as preliminary. Further studies, therefore, should be conducted to confirm these findings. 
Implication of key findings: Lisdexamfetamine is an efficacious stimulant for treating child and adolescent ADHD.

Keywords: lisdexamfetamine, attention-deficit/hyperactivity disorder, meta-analysis, acceptability, tolerability

\section{Background}

Attention-deficit/hyperactivity disorder (ADHD) is a common neurobehavioral problem in children and adolescents with a worldwide prevalence rate of $5 \%-10 \%$ in children and adolescents. ${ }^{1,2}$ Children with ADHD may experience hyperactivity, impulsivity and inattention, while, adolescents with ADHD may have only impulsivity and inattention. Additionally, child and adolescent ADHD tends to have an executive function deficit. ${ }^{3}$ Therefore, those patients have increased risks of difficulties in many aspects of their life such as behavioral and academic problems. $^{3}$

Currently, psychostimulants have been indicated as the first-line treatments in child and adolescent ADHD., Although these active agents have been used effectively for many years, there is a persistent concern for their potential abuse and diversion. ${ }^{6}$ The use of non-stimulants in the treatment of patients with ADHD such as guanfacine may be effective and reduce the potential abuse or diversion. Hence, alternative medication which is comparable in efficacy and tolerability, with less potential abuse and diversion may be beneficial for these patients.

Known as a stimulant, lisdexamfetamine (LDX), an inactive and water-soluble prodrug, after it is absorbed from the gastrointestinal tract, is converted to 1-lysine, an essential amino acid, and dextroamphetamine, which has a therapeutic effect in the reduction of ADHD symptoms. ${ }^{7}$ Since LDX requires an enzyme in red blood cells to be converted to the active form of amphetamine, it tends to have less potential for drug abuse and diversion, and has a relatively long-acting property. ${ }^{8,9}$

Previous studies documented that LDX was an effective, safe, and well-tolerated stimulant in the treatment of ADHD in children and adolescents. ${ }^{10-13}$ Additionally, recent evidence demonstrated its maintenance of long-term efficacy in child and adolescent ADHD. ${ }^{14}$ Although randomized controlled trials (RCTs) showed the efficacy and tolerability of LDX in child and adolescent ADHD, ${ }^{10-13}$ each trial had a limited sample size. A meta-analysis, typically more effective in determining the true effect size, was the method used to assess the efficacy, acceptability, and tolerability of LDX for child and adolescent ADHD.

In this systematic review, one primary aim was to determine the efficacy of LDX versus placebo in the treatment of child and adolescent ADHD. Its acceptability and tolerability were also examined.

\section{Methods}

Because the first papers on LDX were published in PubMed in 2007, the searches of relevant studies were performed from January 2007 to September 2014.

\section{Eligibility criteria}

Any randomized, placebo-controlled trials of LDX conducted in children and adolescents with ADHD spectrum and showing scores of standardized ADHD rating were included. The improvement and discontinuation rates needed to be presented. ADHD spectrum consists of ADHD, attention deficit disorder, hyperkinetic syndrome, and hyperkinetic reaction diagnosed by any set of criteria. However, to be more thorough, the languages of such RCTs were not limited.

\section{Information sources}

Studies, restricted to "humans", presented in the SCOPUS, MEDLINE, CINAHL, Cochrane Controlled Trials Register databases, ClinicalTrials.gov, and EU Clinical Trials Register databases were also searched in September 2014. Besides, references of articles, given by any means, were also searched. The relevant trials included in this meta-analysis were only RCTs or controlled clinical trials.

\section{Database search}

To more sensitively identify the RCTs and controlled clinical trials, the searching technique used a combination of the following words and phrases: [(lisdexamfetamine) OR (Vyvanse) OR (SPD489)] AND [(attention-deficit hyperactivity disorder) OR (ADHD) OR (attention-deficit disorder) OR (hyperkinetic syndrome) OR (hyperkinetic reaction)]. This technique was carried out with all databases.

\section{Study selection}

The abstracts of articles, established from the databases, were independently examined by the authors (NM and BM) for determining whether they were suitable according to the eligibility criteria defined previously. After the full-text versions of relevant articles were provided, the authors then independently examined them. In cases of disagreement, the authors discussed and resolved them by means of consensus. 


\section{Data collection process}

NM extracted the important outcomes into the data extraction form. Then BM reviewed those extracted results, again. All disagreements were also resolved by consensus.

\section{Data items}

The important outcomes extracted and collected from all clinical studies were comprised of the following: 1) the items which were necessary in assessment of the study quality; 2) essential fundamental characteristics of eligible subjects, diagnostic criteria, study design for individual trial and exclusion and inclusion criteria; 3) preparation, dose, and time course of LDX administration; 4) important results applied for synthesis. If possible, the intent-to-treat results were included.

\section{Risk of bias in individual studies}

The internal validity (quality) assessments were carried out by the authors (NM and BM). As stated in the Handbook of Cochrane Collaboration, risks of bias for each trial were evaluated as follows: 1) the generation of randomized sequences, 2) concealment of allocation, 3) blinding, 4) incomplete outcomes, 5) selective reporting, 6) other sources of bias, and 7) baseline similarity. ${ }^{15}$

\section{Summary measures}

The interesting outcomes gathered in this review were composed of efficacy, acceptability, and tolerability. Efficacy outcomes included the pooled mean-endpoint or -change scores measured by the standardized ADHD rating scales, and the pooled rates of improvement assessed by any set of criterion. Although acceptability and tolerability may be used interchangeably, they independently have a specific definition. Based on antecedent systematic reviews, the acceptability, defined as the portion of subjects leaving the study early for any reason (dropout rates) after treatment, ${ }^{16,17}$ was evaluated by the rate of overall discontinuation in this review. ${ }^{18}$ Accordingly, tolerability, defined as proportion withdrawal from study because of adverse events, estimated from the rate of discontinuation due to adverse events was considered as tolerability. ${ }^{19,20}$

\section{Statistical analysis}

As a rule, either weighted mean differences (WMD) or standardized mean differences (SMD) with the 95\% confidence interval $(95 \% \mathrm{CI})$ are used for the synthesis of continuous results. In case of the same outcome rating scales used across clinical trials, the WMD are reasonably applied for comparison or combination of those outcomes. If varied measures were applied, the SMD in which there were no units were alternatively compared or combined. In the present review, the estimation of all continuous data was performed by using either the WMD or the SMD, and relied on the similarity or dissimilarity of the rating scales used in each included trial. If the standard deviation (SD) of mean-endpoint or -change scores for ADHD rating scales was not available from each included article, then, it would be calculated by using any statistical techniques or direct substitution. ${ }^{21}$ An inverse-variance technique was applied for calculation of the pooled mean-endpoint or -change scores with $95 \%$ CIs.

Synthesis of the dichotomous data was carried out by using relative risks (RRs) with $95 \%$ CI. If an RR is one, it means that there is no difference between the two groups. When an RR is less than one, it suggests that such findings are less likely to occur in the experiment group than in the control group. In the case of an RR of more than one, it indicates that the outcomes are more likely to occur in the experiment group than in the control group. For this meta-analysis, the RRs were used to compare the pooled improvement rates, overall discontinuation rates, and discontinuation rates due to adverse events between two groups. All pooled RRs of dichotomous data with $95 \%$ CIs were estimated by using the Mantel-Haenszel technique.

\section{Synthesis of results}

Synthesis of data is being able to apply either a fixed or random effect approach. For included trials that postulate a common effect size, the fixed effect model is reasonably applied. This model is opposite to a random effect model which ignores the variations across various studies. Therefore, it was impossible to presume that those trials were exactly identical. Accordingly, a random effect model was applied to synthesize all results in this meta-analysis.

\section{Statistical software}

In this review, all data synthesis was carried out by using the RevMan 5.1 (The Nordic Cochrane Centre, Copenhagen, Denmark).

\section{Risk of bias across studies}

As a rule, funnel plot is able to examine the publication bias in meta-analysis. The bias can be determined by a simple graph of the intervention effect estimated from individual study against some measure of each study's size or precision. ${ }^{22}$ Hence, a funnel plot would be applied in this meta-analysis. 


\section{Test of heterogeneity}

In a systematic review, a test of heterogeneity is essentially used to measure the similarity of the clinical study results. Our hypothesis assumed that each eligible trial outcome was not the same effect caused by the quality of methodology in individual study. Therefore, the magnitudes of diversity among the study outcomes were calculated. According to the study outcomes shown by graphic display and the use of the test of heterogeneity, the study outcomes were estimated as to whether they had greater differences than would be expected by chance alone. In case $I^{2}$ was $50 \%$ or more, a significant outcome heterogeneity was considered.

\section{Results}

\section{Study selection}

Based on those database searches, 369 citations were collected (SCOPUS $=133$ studies, Medline $=42$ studies, CINAHL $=75$ studies, Cochrane Controlled Trials Register $=87$ studies, ClinicalTrials.gov $=27$ studies and
EU Clinical Trials Register $=5$ studies) (see Figure 1). When the duplicate articles were discarded, the 280 studies were carefully considered. After examining the titles and abstracts of the remaining studies, 264 articles were eliminated because they did not meet the included criteria. Then 16 full articles were carefully inspected. Eleven clinical studies were excluded; six trials were the post hoc analysis of data from previous studies, ${ }^{13,23-27}$ four studies have not reported the study results ${ }^{28-31}$ and one was still recruiting participants ${ }^{32}$ therefore, only five studies ${ }^{10-12,33,34}$ were eligible in the present review. There was no relevant study that met the eligibility criteria.

\section{Study characteristics}

A total of 1,016 children and adolescents with ADHD were included. The duration of five eligible RCTs was 4 to 11 weeks. ${ }^{10-12,33,34}$ Of five RCTs, two were the crossover studies. ${ }^{10,34}$ All studies reported the washout periods (3 days to 6 weeks). The dosage of LDX medication ranged from

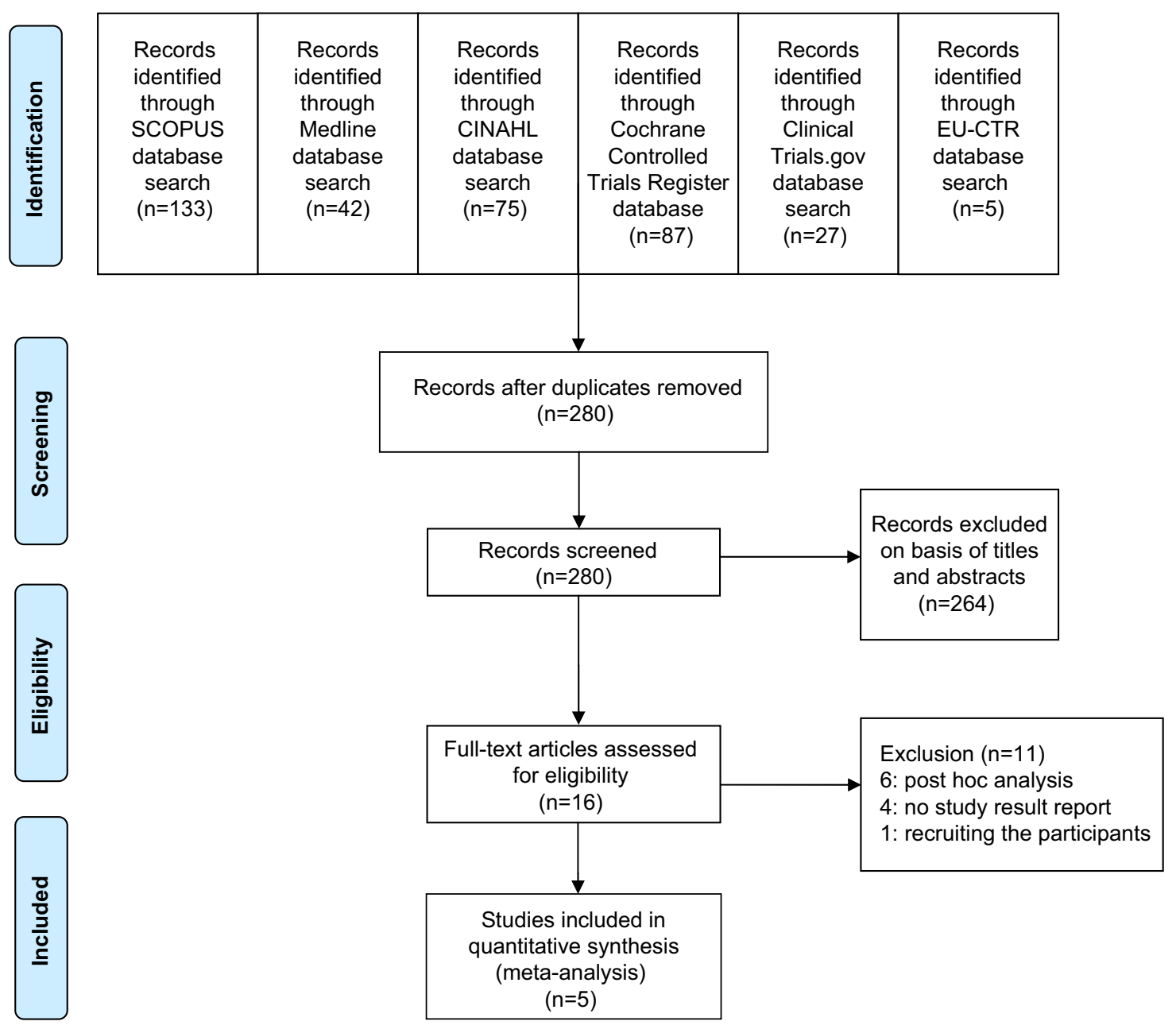

Figure I Flow diagram of study. 


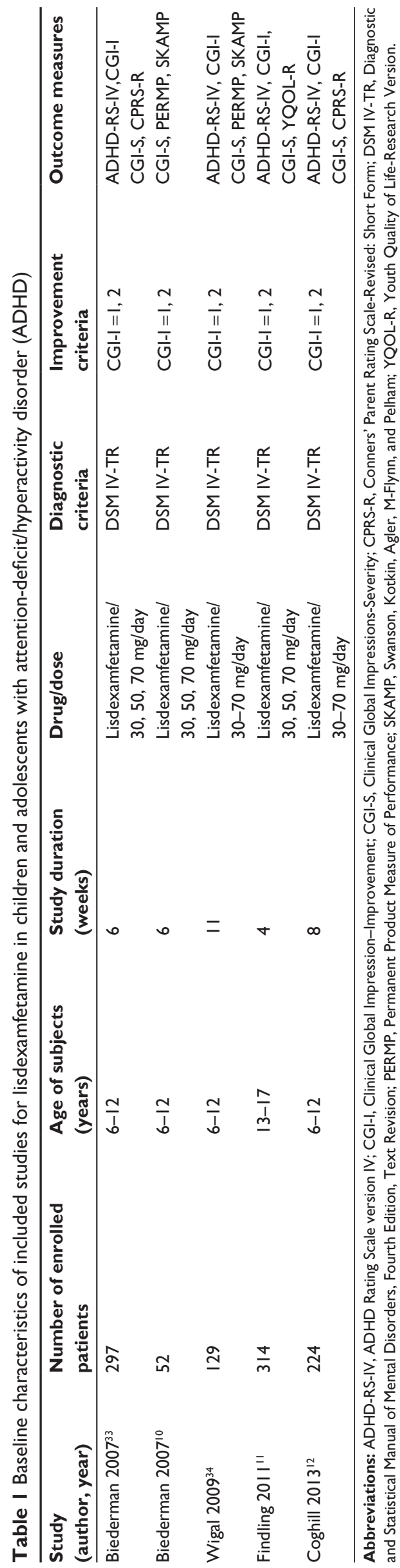

30 to $70 \mathrm{mg} /$ day. The baseline characteristics of included studies are shown in Table 1.

All mean change scores were assessed by using similar rating scales across the included trials, therefore, the WMD were considered in calculating and synthesizing those continuous data. All studies presented the mean change scores of the ADHD Rating Scale Version IV (ADHD-RS-IV), improvement rates, overall discontinuation rates, and discontinuation due to adverse events. However, only two studies ${ }^{11,12}$ reported the mean change score of systolic blood pressure (SBP) and diastolic blood pressure (DBP), and pulse rates.

\section{Risk of bias within studies}

All included clinical studies used the randomized, doubleblind technique. ${ }^{10-12,33,34} \mathrm{An}$ intention-to-treat population was applied for four clinical trials. ${ }^{10,11,33,34} \mathrm{~A}$ sequence generation of randomization and allocation concealment were not reported in two studies. ${ }^{10,34}$ The blinding of outcome assessment and baseline similarity were demonstrated in all studies. However, freedom of selective reporting was determined in three studies (see Table 2). ${ }^{11,12,34}$

\section{Results of individual studies}

Since there were three doses of LDX-treated groups (30, 50 , and $70 \mathrm{mg}$ ), pooled mean change scores (SD) of those outcomes from individual clinical trials were utilized to analyze and synthesize. ${ }^{11,33}$ The mean-changed scores of ADHD-RS-IV for LDX-treated group in each study were significantly greater than that of the placebo-treated group (see Figure 2).The rates of improvement from the individual studies were also significantly different (see Figure 3). There were two studies ${ }^{11,12}$ that reported the mean change scores of the vital signs (SBP, DBP, and pulse rates) of subjects. The mean-changed scores of SBP and DBP of each trial were not significantly different between the two groups (see Figures 4 and 5). However, the mean-changed scores of pulse rates

Table 2 Summary of risk of bias in clinical controlled trials of lisdexamfetamine in child and adolescent attention-deficit/ hyperactivity disorder (ADHD)

\begin{tabular}{llllllll}
\hline Study & \multicolumn{7}{l}{ Issues of bias } \\
\cline { 2 - 7 } (author, year) & I & $\mathbf{2}$ & $\mathbf{3}$ & $\mathbf{4}$ & $\mathbf{5}$ & $\mathbf{6}$ & $\mathbf{7}$ \\
\hline Biederman 200733 & L & L & L & L & U & L & L \\
Biederman 200710 & U & U & L & L & U & L & L \\
Wigal 200934 & U & U & L & L & L & L & L \\
Findling 201 I'1 & L & L & L & L & L & L & L \\
Coghill 201312 & L & L & L & L & L & L & L \\
\hline
\end{tabular}

Notes: I= Adequate sequence generation; $2=$ allocation concealment; $3=$ blinding (subjective outcome); $4=$ dropout data addressed; $5=$ free of selective reporting; $6=$ free of other bias; $7=$ baseline similarity;

Abbreviations: $\mathrm{U}$, unclear; L, low risk of bias. 


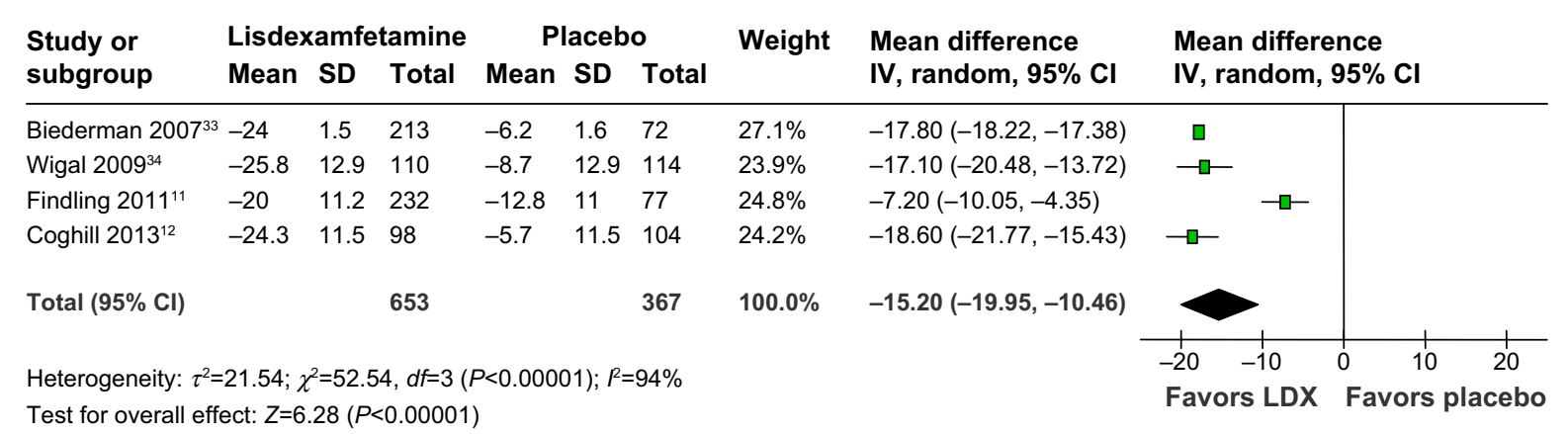

Figure 2 Comparison of the mean change scores of ADHD rating scales ( $95 \%$ confidence interval) in child and adolescent ADHD: lisdexamfetamine versus placebo. Abbreviations: $\mathrm{Cl}$, confidence interval; IV, inverse variance; ADHD, attention-deficit/hyperactivity disorder; LDX, lisdexamfetamine; SD, standard deviation; df, degrees of freedom.

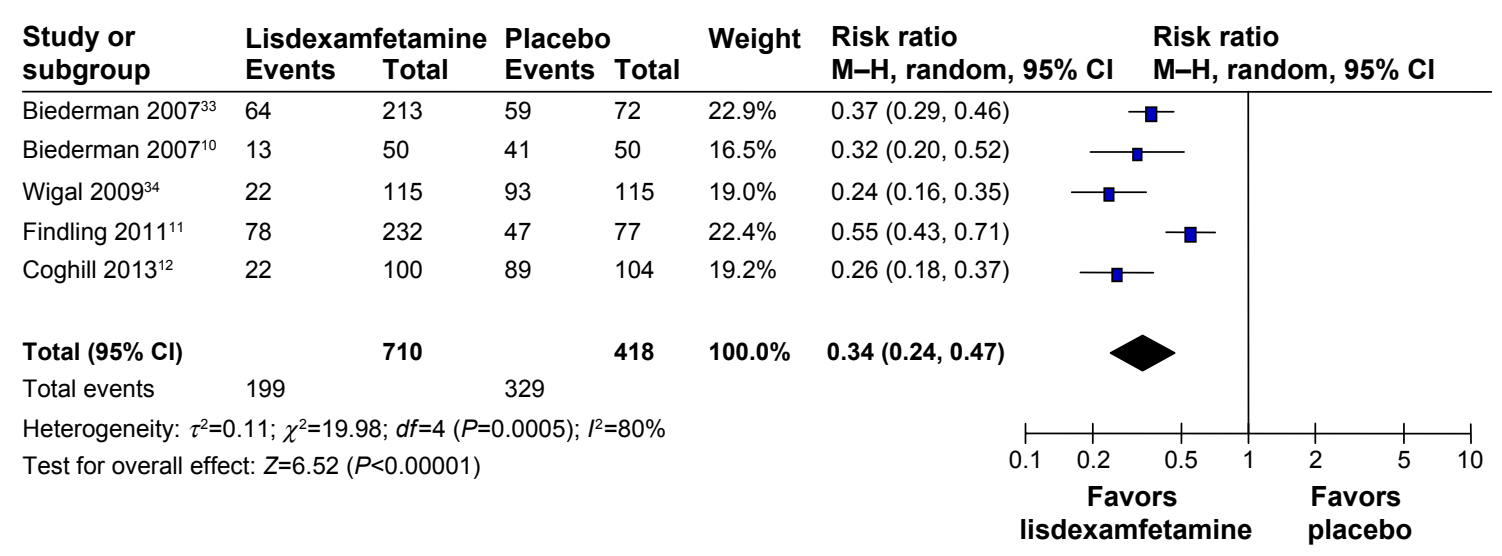

Figure 3 Comparison of relative risk ( $95 \%$ confidence interval) for rates of clinical improvement in child and adolescent ADHD: lisdexamfetamine versus placebo. Abbreviations: ADHD, attention-deficit/hyperactivity disorder; $\mathrm{Cl}$, confidence interval; $d f$, degrees of freedom; LDX, lisdexamfetamine; $M-H$, Mantel-Haenszel.

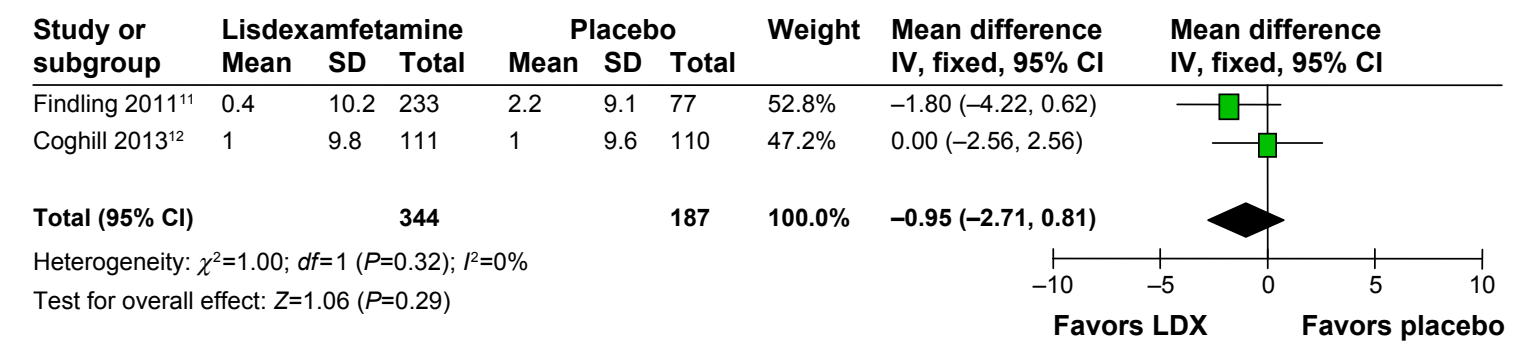

Figure 4 Comparison of the mean change scores of systolic blood pressure and $95 \%$ confidence interval in child and adolescent ADHD: lisdexamfetamine versus placebo. Abbreviations: $\mathrm{Cl}$, confidence interval; IV, inverse variance; ADHD, attention-deficit/hyperactivity disorder; LDX, lisdexamfetamine; SD, standard deviation; df, degrees of freedom.

\begin{tabular}{|c|c|c|c|c|c|c|c|c|c|c|}
\hline \multirow{2}{*}{$\begin{array}{l}\text { Study or } \\
\text { subgroup }\end{array}$} & \multicolumn{3}{|c|}{ Lisdexamfetamine } & \multicolumn{3}{|c|}{ Placebo } & \multirow[t]{2}{*}{ Weight } & \multirow{2}{*}{$\begin{array}{l}\text { Mean difference } \\
\text { IV, random, } 95 \% \mathrm{CI}\end{array}$} & \multirow{2}{*}{$\begin{array}{l}\text { Mean difference } \\
\text { IV, random, } 95 \% \mathrm{CI}\end{array}$} & \\
\hline & Mean & SD & Total & Mean & SD & Total & & & & \\
\hline Findling $2011^{11}$ & 0.2 & 9.6 & 233 & 1.2 & 8.7 & 77 & $47.5 \%$ & $-1.00(-3.30,1.30)$ & & \\
\hline Coghill $2013^{12}$ & 1.1 & 8.1 & 111 & 0.5 & 8.5 & 110 & 52.5 & $0.60(-1.59,2.79)$ & & \\
\hline Total $(95 \% \mathrm{Cl})$ & & & 344 & & & 187 & $100.0 \%$ & $-0.16(-1.75,1.43)$ & & \\
\hline \multicolumn{8}{|c|}{ Heterogeneity: $\tau^{2}=0.00 ; \chi^{2}=0.97 ; d f=1(P=0.32) ; l^{2}=0 \%$} & -10 & 5 & 10 \\
\hline \multicolumn{8}{|c|}{ Test for overall effect: $Z=0.20(P=0.84)$} & \multicolumn{3}{|c|}{ Favors LDX } \\
\hline
\end{tabular}

Figure 5 Comparison of mean change scores of diastolic blood pressure and 95\% confidence interval in child and adolescent ADHD: lisdexamfetamine versus placebo. Abbreviations: $\mathrm{Cl}$, confidence interval; IV, inverse variance; ADHD, attention-deficit/hyperactivity disorder; LDX, lisdexamfetamine; SD, standard deviation; $d f$, degrees of freedom. 


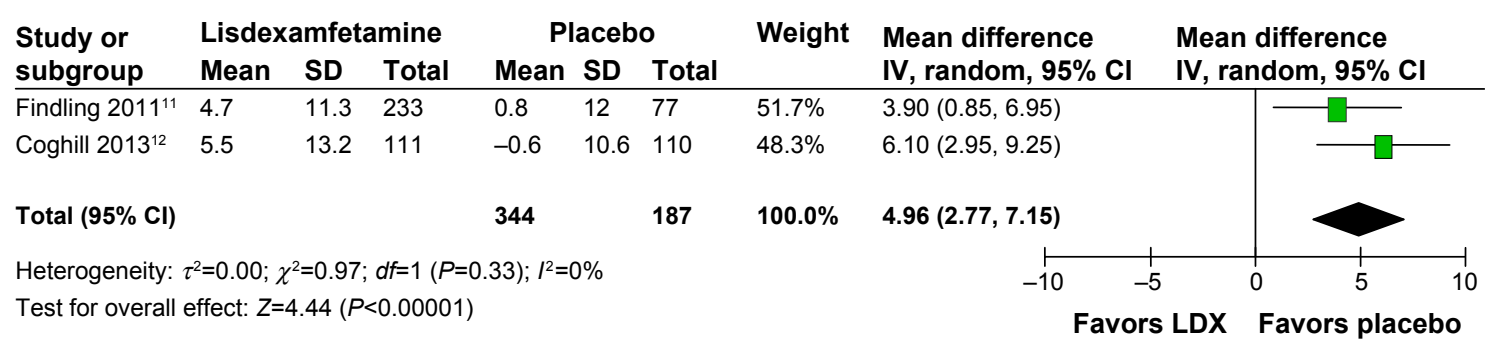

Figure 6 Comparison of the mean change scores of pulse rate and $95 \%$ confidence interval in child and adolescent ADHD: lisdexamfetamine versus placebo. Abbreviations: $\mathrm{Cl}$, confidence interval; IV, inverse variance; ADHD, attention-deficit/hyperactivity disorder; LDX, lisdexamfetamine; SD, standard deviation; $d f$, degrees of freedom.

were significantly different between the two groups (see Figure 6).

\section{Synthesis of results Efficacy}

According to efficacy outcomes, there was significant heterogeneity in the mean-changed ADHD-RS-IV score and improvement rate. Based on synthesis of four studies, ${ }^{11,12,33,34}$ the pooled mean-changed ADHD-RS-IV score of child and adolescent ADHD in the LDX-treated group was significantly greater than that of the placebo-treated group (WMD [95\% CI] of -15.20 [-19.95, -10.46], $I^{2}=94 \%$ ) (see Figure 2).

The pooled improvement rate of child and adolescent ADHD in the LDX-treated groups was significantly higher than that of the placebo-treated group (RR [95\% CI] of 0.34 $[0.24,0.47], I^{2}=80 \%$ ) (see Figure 3 ). According to the pooled improvement rate, the number needing treatment $(95 \% \mathrm{CI})$ was $1.97(1.80,2.21)$.

\section{Vital signs}

Based on the mean-changed vital sign scores, there was not significant heterogeneity. The pooled mean-changed SBP and DBP scores of child and adolescent ADHD between the LDX- and placebo-treated group had no significant differences with WMD $(95 \% \mathrm{CI})$ of $-0.95(-2.71,0.81), I^{2}=0 \%$ and $\operatorname{WMD}(95 \% \mathrm{CI})$ of $0.16(-1.75,1.43), I^{2}=0 \%$, respectively (see Figures 4 and 5). Unfortunately, the pooled mean-changed pulse rate score of child and adolescent ADHD between the LDX- and placebo-treated group was significantly different (WMD [95\% CI] of $4.96[2.77,7.15], I^{2}=0 \%$ ) (see Figure 6).

\section{Discontinuation rates (acceptability and tolerability)}

Significant heterogeneity was found in the overall discontinuation rate. The pooled overall discontinuation rate (acceptability) and discontinuation rate due to adverse events (tolerability) in child and adolescent ADHD between the
LDX- and placebo-treated groups had no significant differences with RR ( $95 \% \mathrm{CI})$ of $0.78(0.46,1.31), I^{2}=63 \%$ and RR $(95 \% \mathrm{CI})$ of $1.99(0.70,5.64), I^{2}=16 \%$, respectively.

\section{Risk of bias across studies}

As a rule, the funnel plot asymmetry is able to estimate the publication bias in the meta-analysis which includes at least ten studies. In this review, only five RCTs were included. Accordingly, the real chance asymmetry was difficult to distinguish, ${ }^{22}$ therefore, this meta-analysis did not apply the funnel plot. However, two studies had the lack of clearness of a risk of bias in two or more domains (see Table 2). ${ }^{10,34}$

\section{Discussion}

In the present review, five RCTs of LDX versus placebo in the treatment of child and adolescents were found eligible. This review suggests that LDX is more efficacious than the placebo in the treatment of child and adolescent ADHD. The pooled improvement rate of LDX versus placebo treatments was $72 \%$ and $21 \%$, respectively. Based on the pooled improvement rate, the number needing treatment of 2 indicated that one in every two children and adolescents with ADHD will benefit from LDX treatment. Although LDX as compared with placebo did not increase SBP and DBP, it did increase the pulse rates in children and adolescents with ADHD. Based on discontinuation rates, the acceptability and tolerability of LDX treatment were not better than that of the placebo. However, its tolerability, measured by the discontinuation rate due to adverse events, was comparable to the placebo. Considering these findings, LDX has efficacy, acceptability, and tolerability in treatment of child and adolescent ADHD.

Efficacy of LDX in the treatment of child and adolescent ADHD from this review was similar to other stimulants. Several studies have shown the efficacy of stimulants in the treatment of child and adolescent ADHD, including Ritalin ${ }^{\circledR}$ LA, 
a modified-release formulation of methylphenidate (MPH), ${ }^{35}$ osmotic-release oral system of MPH (OROS MPH), ${ }^{36,37}$ and mixed amphetamine salts extended release (Adderall XR). ${ }^{38}$ Considered as a rate of clinical improvement, this review suggests that $72 \%$ of children and adolescents with ADHD in the LDX-treated group compared with $21 \%$ of the placebotreated group was categorized in "much improved" or "very much improved" as measured by the Clinical Global Impression improvement subscale score, which was similar to a previous study in adolescents with ADHD treated with the OROS MPH (OROS MPH: 52\% versus placebo: $31 \%$ ). ${ }^{39}$ The efficacy of LDX in such patients is explained by its active metabolite, dextroamphetamine. Like other stimulants, dextroamphetamine blocks the reuptake of norepinephrine and dopamine into the presynaptic neuron and directly increases the release of these monoamines extraneuronally. ${ }^{9}$

Cardiovascular effect is another concern in the use of stimulants in the treatment of child and adolescent ADHD. There was a study of the short-term cardiovascular effects of MPH and Adderall. The study found that changes in the SBP, DBP, and pulse rates with both medications were not clinically significant. ${ }^{40}$ Another study of cardiovascular effects suggested that no clinical changes occurred in blood pressure and pulse rates in school-aged children with ADHD treated with mixed amphetamine salts extended release. ${ }^{41}$ In the present review, although there were not significant changes in blood pressure from baseline in LDX treated subjects compared with the placebo group, which was similar to the previous studies of stimulant treatment in child and adolescent ADHD, there were significant changes in pulse rates in the LDX treated group from baseline, compared with placebo. Hence, cautious use of LDX treatment in child and adolescent ADHD is recommended.

Based on the present review, the acceptability of LDX treatment in child and adolescent ADHD was comparable to the placebo, which was similar to LDX treatment in adult ADHD. According to a recent review, the acceptability of LDX treatment in adult ADHD was comparable to the placebo (RR [95\% $\mathrm{CI}]$ of $0.82[0.59,1.14]) .{ }^{42} \mathrm{In}$ addition, a systematic review also showed that acceptability of MPH and atomoxetine treatments in child and adolescent ADHD was comparable. ${ }^{43}$

In the present review, the tolerability of LDX in the treatment of child and adolescent ADHD was comparable to the placebo. Similarly, a recent review in adult ADHD also found that those patients tolerated LDX treatment well. ${ }^{42}$ Unfortunately, a recent review of MPH treatment in adult ADHD demonstrated that its tolerability was lower than that of placebo. ${ }^{44}$ Good tolerability of LDX may be explained by the prodrug formulation which may reduce the adverse events noted in other psychostimulants while its acceptability may be explained by its metabolite, dextroamphetamine, which is efficacious for ADHD treatment.

Although acceptability, tolerability, and blood pressure change in LDX treatment were comparable to the placebo, there was a concern of cardiomyopathy in LDX users. Three in 5,422 persons who reported adverse events while taking LDX had cardiomyopathy; one male adult and two male teenagers. ${ }^{45}$ Even if the rate of incidence is rare but serious, further studies are essential to determine the potential adverse events.

Several limitations were observed in the present review. Firstly, a small number of eligible studies (five RCTs) were included which may reduce the potential impact of the systematic review. Hence, cautious interpretation of these outcomes is advised. Secondly, the five included studies were financially supported by a pharmaceutical company holding the patent of LDX, consequently, these findings should be carefully interpreted. Finally, publication bias cannot be calculated in the present review, since there was such a small number of eligible trials. ${ }^{22}$

\section{Conclusion}

According to the findings of this review, LDX is effective, and well-tolerated in the treatment of child and adolescent ADHD. Unfortunately, its acceptability is no greater than a placebo. Due to limited included studies, this outcome should be cautiously interpreted. Further studies may be warranted by these findings.

\section{Authors' contributions}

All authors conceived the idea, prepared the study protocol, and approved the manuscript in its current form, analyzed the data and prepared the manuscript. NM and BM searched the databases and extracted the data.

\section{Disclosure}

This review received financial support from Chiang Mai University, Thailand.

$\mathrm{BM}$ has received honoraria and/or travel reimbursement from Lundbeck and Pfizer. NM has received travel reimbursement from Servier, Lundbeck and Pzifer. SL has no conflict of interest. SS has received honoraria and/or research grants from Janssen-Cilag, Thai-Otsuka, Lundbeck, and AstraZeneca. AN has no conflict of interest. MS has received honoraria, consultancy fees, research grants, and/or travel reimbursement from AstraZeneca, GlaxoSmithKline, Pfizer, Janssen-Cilag, Johnson \& Johnson, Lundbeck, Thai-Otsuka, Sanofi-Aventis, and Servier. PW has no conflict of interest. 


\section{References}

1. Montiel C, Pena JA, Montiel-Barbero I, Polanczyk G. Prevalence rates of attention deficit/hyperactivity disorder in a school sample of Venezuelan children. Child Psychiatry Hum Dev. 2008;39(3):311-322.

2. Polanczyk G, Rohde LA. Epidemiology of attention-deficit/hyperactivity disorder across the lifespan. Curr Opin Psychiatry. 2007;20(4): 386-392.

3. Biederman J, Monuteaux MC, Doyle AE, et al. Impact of executive function deficits and attention-deficit/hyperactivity disorder (ADHD) on academic outcomes in children. J Consult Clin Psychol. 2004;72(5): 757-766.

4. Koneski JA, Casella EB, Agertt F, Ferreira MG. Efficacy and safety of methylphenidate in treating ADHD symptoms in children and adolescents with uncontrolled seizures: a Brazilian sample study and literature review. Epilepsy Behav. 2011;21(3):228-232.

5. Maneeton N, Maneeton B, Intaprasert S, Woottiluk P. A systematic review of randomized controlled trials of bupropion versus methylphenidate in the treatment of attention-deficit/hyperactivity disorder. Neuropsychiatr Dis Treat. 2014;10:1439-1449.

6. Clemow DB, Walker DJ. The potential for misuse and abuse of medications in ADHD: a review. Postgrad Med. 2014;126(5):64-81.

7. Blick SK, Keating GM. Lisdexamfetamine. Paediatr Drugs. 2007;9(2): 129-135; discussion 136-138.

8. Elbe D, Macbride A, Reddy D. Focus on Lisdexamfetamine: A Review of its use in Child and Adolescent Psychiatry. J Can Acad Child Adolesc Psychiatry. 2010;19(4):303-314.

9. Madaan V, Kolli V, Bestha DP, Shah MJ. Update on optimal use of lisdexamfetamine in the treatment of ADHD. Neuropsychiatr Dis Treat. 2013;9:977-983.

10. Biederman J, Boellner SW, Childress A, Lopez FA, Krishnan S, Zhang Y. Lisdexamfetamine dimesylate and mixed amphetamine salts extended-release in children with ADHD: a double-blind, placebo-controlled, crossover analog classroom study. Biol Psychiatry. 2007;62(9):970-976.

11. Findling RL, Childress AC, Cutler AJ, et al. Efficacy and safety of lisdexamfetamine dimesylate in adolescents with attention-deficit/hyperactivity disorder. J Am Acad Child Adolesc Psychiatry. 2011;50(4):395-405.

12. Coghill D, Banaschewski T, Lecendreux M, et al. European, randomized, phase 3 study of lisdexamfetamine dimesylate in children and adolescents with attention-deficit/hyperactivity disorder. Eur Neuropsychopharmaco. 2013;23(10):1208-1218.

13. Coghill DR, Banaschewski T, Lecendreux M, et al. Efficacy of lisdexamfetamine dimesylate throughout the day in children and adolescents with attention-deficit/hyperactivity disorder: results from a randomized, controlled trial. Eur Child Adolesce Psychiatry. 2014;23(2):61-68.

14. Coghill DR, Banaschewski T, Lecendreux M, et al. Maintenance of efficacy of lisdexamfetamine dimesylate in children and adolescents with attention-deficit/hyperactivity disorder: randomized-withdrawal study design. J Am Acad Child Adolesc Psychiatry. 2014;53(6):647-657.

15. Higgins JPT, Altman DG. Assessing risk of bias in included studies. In: Higgins JPT, Green S, editors. Cochrane Handbook for Systematic Reviews of Interventions. Version 5.1.0 (Updated March 2011). The Cochrane Collaboration; 2009.

16. Koesters M, Guaiana G, Cipriani A, Becker T, Barbui C. Agomelatine efficacy and acceptability revisited: systematic review and meta-analysis of published and unpublished randomised trials. Br J Psychiatry. 2013; 203(3):179-187.

17. Okumura Y, Ichikura K. Efficacy and acceptability of group cognitive behavioral therapy for depression: a systematic review and metaanalysis. J Affect Disord. 2014;164:155-164.

18. Cipriani A, Furukawa TA, Salanti G, et al. Comparative efficacy and acceptability of 12 new-generation antidepressants: a multipletreatments meta-analysis. Lancet. 2009;373(9665):746-758.

19. Papakostas GI. Tolerability of modern antidepressants. J Clin Psychiatry. 2008;69 Suppl E1:8-13.

20. Baldwin D, Woods R, Lawson R, Taylor D. Efficacy of drug treatments for generalised anxiety disorder: systematic review and meta-analysis. BMJ. 2011;342:d1199.
21. Wiebe N, Vandermeer B, Platt RW, Klassen TP, Moher D, Barrowman NJ. A systematic review identifies a lack of standardization in methods for handling missing variance data. J Clin Epidemiol. 2006;59(4): 342-353.

22. Sterne JAC, Egger M, Moher D. Addressing Reporting Biases. In: Higgins JPT, Green S, editors. Cochrane Handbook for Systematic Reviews of Interventions. Version 5.1.0 (Updated March 2011). The Cochrane Collaboration; 2009.

23. Lopez FA, Ginsberg LD, Arnold V. Effect of lisdexamfetamine dimesylate on parent-rated measures in children aged 6 to 12 years with attention-deficit/hyperactivity disorder: a secondary analysis. Postgrad Med. 2008;120(3):89-102.

24. Wigal SB, Kollins SH, Childress AC, Adeyi B. Efficacy and tolerability of lisdexamfetamine dimesylate in children with attention-deficit/ hyperactivity disorder: sex and age effects and effect size across the day. Child Adolesc Psychiatry Ment Health. 2010;4:32.

25. Soutullo C, Banaschewski T, Lecendreux M, et al. A post hoc comparison of the effects of lisdexamfetamine dimesylate and osmotic-release oral system methylphenidate on symptoms of attention-deficit hyperactivity disorder in children and adolescents. CNS Drugs. 2013;27(9): 743-751.

26. Jain R, Babcock T, Burtea T, et al. Efficacy and safety of lisdexamfetamine dimesylate in children with attention-deficit/hyperactivity disorder and recent methylphenidate use. Adv Ther. 2013;30(5): 472-486.

27. Childress AC, Arnold V, Adeyi B, et al. The effects of lisdexamfetamine dimesylate on emotional lability in children 6 to 12 years of age with ADHD in a double-blind placebo-controlled trial. J Atten Disord. 2014; 18(2):123-132.

28. Clinical Study Centers, LLC. Effect of Vyvanse on sleep in children aged 6-12 years with attention deficit hyperactivity disorder (ADHD). Available from: http://clinicaltrials.gov/show/NCT00807222. NLM identifier: NCT00807222. Accessed September 27, 2014.

29. Shire. Effectiveness of Vyvanse compared to concerta in adolescents with attention-deficit/hyperactivity disorder. Available from: http:// clinicaltrials.gov/ct2/show/record/NCT01552902. NLM identifier: NCT01552902. Accessed September 27, 2014.

30. Shire. Effectiveness of Vyvanse compared to concerta in adolescents with attention-deficit/hyperactivity disorder. Available from: http:// clinicaltrials.gov/ct2/show/study/NCT01552915. NLM identifier: NCT01552915. Accessed September 27, 2014.

31. Shire. Lisdexamfetamine dimesylate 2-year safety study in children and adolescents with attention-deficit/hyperactivity disorder (ADHD). Available from: http://clinicaltrials.gov/ct2/show/study/NCT01328756. NLM identifier: NCT01328756. Accessed September 27, 2014.

32. University of California, Davis. Lisdexamfetamine's effect in ADHD in the brain and cognition. Available from: http://clinicaltrials.gov/ ct2/show/NCT02170298. NLM identifier: NCT02170298. Accessed September 27, 2014.

33. Biederman J, Krishnan S, Zhang Y, McGough JJ, Findling RL. Efficacy and tolerability of lisdexamfetamine dimesylate (NRP-104) in children with attention-deficit/hyperactivity disorder: a phase III, multicenter, randomized, double-blind, forced-dose, parallel-group study. Clin Ther. 2007;29(3):450-463.

34. Wigal SB, Kollins SH, Childress AC, Squires L, Study G. A 13-hour laboratory school study of lisdexamfetamine dimesylate in school-aged children with attention-deficit/hyperactivity disorder. Child Adolesc Psychiatry Ment Health. 2009;3(1):17.

35. Schulz E, Fleischhaker C, Hennighausen K, et al. A double-blind, randomized, placebo/active controlled crossover evaluation of the efficacy and safety of Ritalin (R) LA in children with attention-deficit/ hyperactivity disorder in a laboratory classroom setting. J Child Adolesc Psychopharmacol. 2010;20(5):377-385.

36. Murray DW, Childress A, Giblin J, Williamson D, Armstrong R, Starr HL. Effects of OROS methylphenidate on academic, behavioral, and cognitive tasks in children 9 to 12 years of age with attention-deficit/ hyperactivity disorder. Clin Pediatr (Phila). 2011;50(4):308-320. 
37. Wigal SB, Wigal T, Schuck S, et al. Academic, behavioral, and cognitive effects of OROS(R) methylphenidate on older children with attentiondeficit/hyperactivity disorder. J Child Adolesc Psychopharmacol. 2011; 21(2):121-131.

38. Spencer TJ, Wilens TE, Biederman J, Weisler RH, Read SC, Pratt R. Efficacy and safety of mixed amphetamine salts extended release (Adderall XR) in the management of attention-deficit/hyperactivity disorder in adolescent patients: a 4-week, randomized, double-blind, placebocontrolled, parallel-group study. Clin Ther. 2006;28(2):266-279.

39. Wilens TE, McBurnett K, Bukstein O, et al. Multisite controlled study of OROS methylphenidate in the treatment of adolescents with attentiondeficit/hyperactivity disorder. Arch Pediatr Adolesc Med. 2006;160(1): 82-90.

40. Findling RL, Short EJ, Manos MJ. Short-term cardiovascular effects of methylphenidate and adderall. J Am Acad Child Adolesc Psychiatry. 2001;40(5):525-529.

41. Donner R, Michaels MA, Ambrosini PJ. Cardiovascular effects of mixed amphetamine salts extended release in the treatment of school-aged children with attention-deficit/hyperactivity disorder. Biol Psychiatry. 2007;61(5):706-712.
42. Maneeton N, Maneeton B, Suttajit S, Reungyos J, Srisurapanont M, Martin SD. Exploratory meta-analysis on lisdexamfetamine versus placebo in adult ADHD. Drug Des Devel Ther. 2014;8:1685-1693.

43. Hanwella R, Senanayake M, de Silva V. Comparative efficacy and acceptability of methylphenidate and atomoxetine in treatment of attention deficit hyperactivity disorder in children and adolescents: a meta-analysis. BMC Psychiatry. 2011;11:176.

44. Castells X, Cunill R, Capella D. Treatment discontinuation with methylphenidate in adults with attention deficit hyperactivity disorder: a meta-analysis of randomized clinical trials. Eur J Clin Pharmacol. 2013;69(3):347-356.

45. ehealthme.com [homepage on the Internet]. Could Vyvanse cause Stress cardiomyopathy? Personalized health information and community. Available from: http://www.ehealthme.com/ds/vyvanse/ stress+cardiomyopathy. Accessed May 25, 2014.

\section{Publish your work in this journal}

Drug Design, Development and Therapy is an international, peerreviewed open-access journal that spans the spectrum of drug design and development through to clinical applications. Clinical outcomes, patient safety, and programs for the development and effective, safe, and sustained use of medicines are a feature of the journal, which has also been accepted for indexing on PubMed Central. The manuscript management system is completely online and includes a very quick and fair peer-review system, which is all easy to use. Visit http://www.dovepress.com/testimonials.php to read real quotes from published authors.

Submit your manuscript here: http://www.dovepress.com/drug-design-development-and-therapy-journal 